\title{
Posterior Interosseus Nerve Palsy Resulting From Inflammatory Myofibroblastic Pseudotumor: Case Presentation
}

\author{
Rushna Ali, MD, Lynda J.-S. Yang, MD, PhD, P. Troy Henning, DO
}

Local compression by a mass lesion is a potential cause of posterior interosseous nerve (PIN) palsy. Reported cases of PIN pathology do not include inflammatory myofibroblastic pseudotumor. We report the case of a 44-year-old woman with a 3-month history of progressive weakness of the left finger extensors. Sonographic imaging identified a mass compressing the PIN, and histologic examination of the specimen revealed an inflammatory myofibroblastic pseudotumor. Complete resection of the mass while sparing the nerve was possible, and the patient has regained functional use of the left hand. The differential diagnosis of PIN palsy should include inflammatory myofibroblastic pseudotumor.

PM R 2014;6:659-661

\section{INTRODUCTION}

The initial symptoms of posterior interosseous nerve (PIN) pathology may be subtle and mistaken for another process, for example, lateral elbow tendinopathy. The characteristic clinical presentation includes paresis of the finger and thumb extension, thumb abduction, and weakness of the wrist extension through ulnar deviation. Early cases may present as pain localized to the proximal lateral forearm. PIN palsy results from both mechanical and nonmechanical causes. The former process includes local compression under the tendinous arch around the leading edge of the supinator (arcade of Fröhse) or extensor carpi radialis brevis muscles, vessel loops off the adjacent recurrent radial artery, parosteal lipomas, nerve sheath tumors, bicipitoradial bursal effusions, ganglion cysts, mal-united fractures, trauma, or iatrogenic injuries $[1,2]$. The latter includes synovitis related to rheumatoid arthritis, vasculitis, or drug toxicity [3].

Imaging modalities, including magnetic resonance imaging and/or ultrasonography, can be used to assess for entrapment and/or compressive neuropathies around the elbow [4]. Electrodiagnostic studies can be used to further characterize, localize, and help prognosticate the neurologic outcome [5]. No previous report has described a case of PIN injury caused by inflammatory myofibroblastic pseudotumor, a heterogeneous group of benign tumor-like lesions that consist of a chronic inflammatory process associated with reactive proliferation of the mesenchymal tissue with collagen deposits [6,7]. We describe the first reported case, to our knowledge, of PIN palsy that resulted from inflammatory myofibroblastic pseudotumor.

\section{CASE PRESENTATION}

A 44-year-old woman initially presented to her primary physician with a 2-month history of left lateral elbow pain and numbness along the dorsal aspect of the thumb. Results of the initial examination revealed pain with resisted wrist extension and tenderness along the lateral epicondyle. She was initially diagnosed with lateral elbow tendinopathy and was referred to occupational therapy. Due to a lack of improvement and progression of symptoms after 1 month, the patient was referred to the physical medicine and rehabilitation hand clinic. Upon presentation to our clinic, examination revealed minimal tenderness to palpation along the left lateral epicondyle, weakness of thumb

R.A. Department of Neurosurgery, University of Michigan, Ann Arbor, Ml Disclosure: nothing to disclose

L.J.-S.Y. Department of Neurosurgery, University of Michigan, Ann Arbor, Ml Disclosure outside this publication: consultancy, Food and Drug Administration-Center for Devices and Radiological Health

P.T.H. Department of Physical Medicine and Rehabilitation, University of Michigan, $325 \mathrm{E}$. Eisenhower Parkway, Suite 100, Ann Arbor, Ml 48108. Address correspondence to: P.T.H.; e-mail: ptroy@med.umich.edu Disclosure: nothing to disclose

Submitted for publication September 3, 2013; accepted December 28, 2013. 
abduction-extension, finger extension at the metacarpophalangeal joints, and wrist extension through ulnar deviation. The remainder of the motor-sensory examination was normal, as were tendon reflexes of the bilateral upper extremities. The patient underwent evaluation of the lateral elbow region via an M Turbo ultrasound unit with a 13-6 $\mathrm{MHz}$ linear array transducer (Sonosite Inc, Bothell, WA). The lateral elbow tendons, collateral ligaments, and underlying elbow joint were normal. The radial nerve was evaluated proximal to the elbow to the exit of the PIN at the supinator muscle. Proximal to the supinator muscle and displacing the PIN, a well-circumscribed homogeneous hypoechoic mass that measured $2.15 \mathrm{~cm}$ mediolateral $\times$ $1.38 \mathrm{~cm}$ anteroposterior $\times 2.6 \mathrm{~cm}$ proximal to distal was visualized (Figure 1). One week later, an electrodiagnostic study revealed normal radial sensory and median ulnar sensorimotor nerve conduction. Results of needle examination of the triceps, brachioradialis, and flexor carpi radialis were normal. Increased insertional activity and widened polyphasic motor unit action potentials with reduced recruitment were seen within the extensor digitorum communis and indicis.

The patient elected to delay surgery for 3 months. She then underwent surgical resection of the mass, along with neurolysis of the radial nerve and its branches. A welldefined mass was found adjacent to the PIN and was completely resected (Figure 2). Histology revealed a lowgrade spindle-celled lesion consistent with inflammatory myofibroblastic tumor. Follow-up examinations at 1 and 4 months showed gradual improvement in strength and restoration of function.

\section{DISCUSSION}

To our knowledge, this is the first report of PIN palsy related to an inflammatory myofibroblastic tumor. In 1905, Guillain and Courtellemont first reported an isolated PIN palsy attributed to the repetitive trauma of alternating forearm pronation and supination in an orchestra conductor [8].

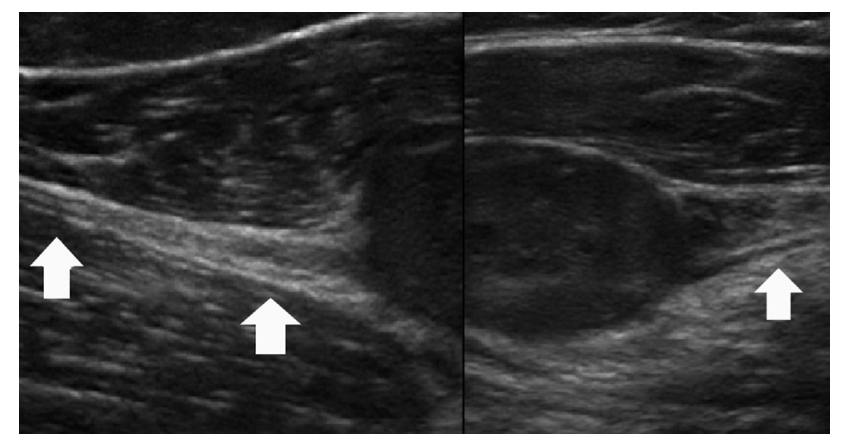

Figure 1. Ultrasound images using a 13-6 $\mathrm{MHz}$ linear array transducer; arrows point to the posterior interosseous nerve. Left $=$ proximal, right $=$ distal, top $=$ superficial, bottom $=$ deep.

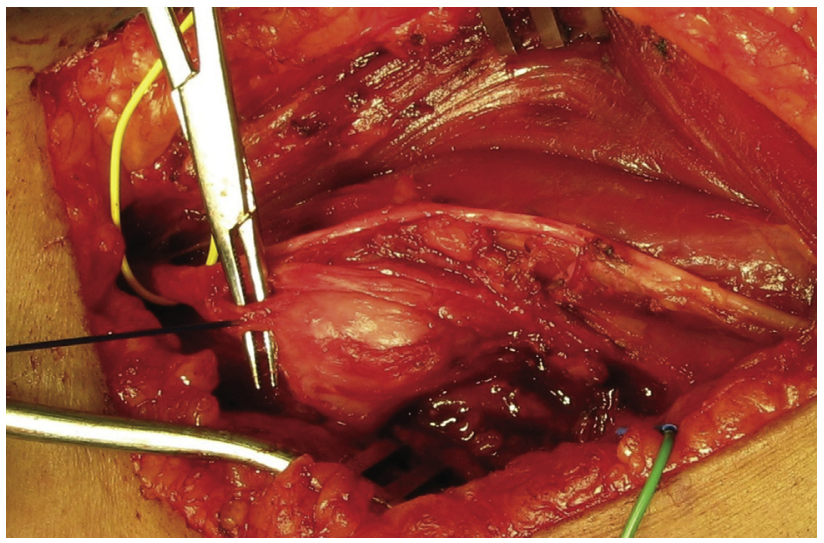

Figure 2. Intraoperative view of the lesion with yellow vesselloop around the posterior interosseous nerve.

Other causes of PIN palsy comprise mechanical and nonmechanical etiologies. Most commonly, the PIN is compressed along the leading edge of the supinator muscle at the arcade of Fröhse [2]. Other compressive lesions include fibrous bands at the level of the radial head, lipomas, bicipitoradial bursal effusions, ganglion cyst arising from the anterior capsule of the proximal radioulnar joint, neurofibromas, schwannomas, and synovial hemangiomas $[1,2]$.

Examination of a patient with PIN palsy reveals the loss of active extension at the metacarpophalangeal joints and thumb as well as weakened wrist extension through ulnar deviation. Concurrent wasting of the extensor compartment muscles of the forearm may be present. The sensory and reflex examinations should be normal. Our patient initially reported numbness along the dorsal thumb along with the pain about the lateral elbow. On our examination, her sensation was normal, as was the radial sensory nerve conduction study. Her initial presenting symptoms of lateral elbow pain and paresthesias likely represent a mild form of radial sensory neuropraxia that resolved before her presentation to our clinic. The mass was immediately adjacent to the PIN and deep; this likely explains why this branch was affected to a greater extent than was the superficial radial nerve.

The etiology of inflammatory myofibroblastic pseudotumors are still unknown. Neoplastic processes, local chronic ischemic conditions, and protracted inflammatory reactions to Epstein-Barr virus have been suggested as potential causes in their evolution [7]. Most reports describe tumors that arise within the lung, mesentery, and omentum [7]. Peripheral nerve involvement is exceedingly rare, with 6 case reports that involved cranial nerves, and 1 case each that involved sciatic, median, ulnar, and radial nerves [7]. To date, no report exists of this tumor involving the PIN.

Before a histologic evaluation of the mass, these lesions can mimic other processes, such as schwannomas, neurofibromas, peripheral nerve sheath tumors, amyloid pseudotumors, primary lymphomas, tuberculoid leprous neuropathy, and 
hypertrophic inflammatory neuropathies [6]. Ideally, the mass can be resected while sparing the adjacent nerve as much as possible. One prior publication reported regrowth of the lesion that affected the proximal and distal nerve stumps 2 years after initial resection [6]. In contrast, in a separate case with only partial resection of the most affected nerve fascicles, no tumor regrowth was found on annual contrast-enhanced magnetic resonance imaging studies over an 8-year follow-up period [6]. No evidence exists in the published literature for the use of chemotherapeutic agents or radiation therapy [7].

To be complete, nonsurgical management of other forms of PIN palsy may include a trial of activity modification, stretching, and anti-inflammatory medications because some injuries spontaneously resolve. If, after 3 months of delayed recovery, or if there is progression of neurologic impairment, Fernandez et al [2] recommend decompression of the nerve at the common impingement sites (ie, arcade of Fröhse) as well as removal of compressive lesions (eg, mass, cysts) [2]. To highlight the postoperative outcomes of more common causes of PIN palsy, a 1996 study by Hashizume et al [1] reported on a case series of patients treated for nontraumatic (included focal compression at arcade of Fröhse, lipoma, ganglion cyst, and Parsonage Turner syndrome) posterior interosseous nerve injury [1]. Patients underwent decompression of the PIN and removal of compressive lesions if present at a mean of 9 weeks (2-24 weeks) after onset of paralysis. Twenty-four of 25 patients had normalization of strength at a mean of 4.2 months after surgery.

Although our patient's particular mass was not a part of this case series, the expansion of the inflammatory myofibroblastic pseudotumor likely caused a compressive neuropathy similar to that seen in some of the patients in the study by Hashizume et al [1]. As a result, there is the potential for a good outcome in our patient, because she was treated within a similar time frame and has already shown improvement in function after surgery.

\section{CONCLUSION}

An inflammatory myofibroblastic pseudotumor is a benign process that can compress the posterior interosseous nerve and should be considered as a potential etiology of PIN palsy. Surgical resection of the mass while sparing the adjacent nerve as much as possible is the preferred treatment approach. Histologic assessment helps to confirm the diagnosis of the mass.

\section{REFERENCES}

1. Hashizume H, Nishida K, Nanba Y, Shigeyama Y, Inoue H, Morito Y Non-traumatic paralysis of the posterior interosseous nerve. J Bone Joint Surg 1996;78:771-776.

2. Fernandez E, Pallini R, Lauretti L, Scogna A, Di Rienzo A. Neurosurgery of the peripheral nervous system: The posterior interosseous nerve syndrome. Surg Neurol 1998;49:637-639.

3. Chan JKK, Kennett R, Smith G. Posterior interosseous nerve palsy in rheumatoid arthritis: Case report and literature review. Br J Plastic Surg 2009;62:e556-e560.

4. Chien A, Jamadar D, Jacobson JA, Hayes C, Louis D. Sonography and MR imaging of posterior interosseous nerve syndrome with surgical correlation. AJR Am J Roentgenol 2003;181:219-221.

5. Bevelaqua A-C, Hayter CL, Feinberg JH, Rodeo SA. Posterior interosseous neuropathy: Electrodiagnostic evaluation. HSS J 2012;8:184-189.

6. Perez-Lopez C, Gutierrez M, Isla A. Inflammatory pseudotumor of the median nerve. J Neurosurg 2001;95:124-128.

7. Schitenhelm J, Kaminsky J, Meyermann R, Mittelbronn M. Inflammatory myofibroblastic tumor of the ulnar nerve. J Neurosurg 2007;106: 1075-1079.

8. Dang AC, Rodner CM. Unusual compression neuropathies of the forearm, part 1: Radial nerve. J Hand Surg Am 2009;34:1906-1914. 